\title{
Competencia digital transversal en la formación del profesorado, análisis de una experiencia
}

\section{Transversal digital competence in teacher training: analysis of an experience}

\author{
Diana Marín Suelves ${ }^{1}$, María Isabel Vidal Esteve², José Peirats Chacón ${ }^{3}$, Ángel San Martín \\ Alonso $^{4}$ \\ ${ }^{1}$ Departamento Didáctica y Organización Escolar, Universitat de València, España (diana.marin@uv.es) \\ ${ }^{2}$ Departamento Didáctica y Organización Escolar, Universitat de València, España (isabel.vidal@uv.es) \\ ${ }^{3}$ Departamento Didáctica y Organización Escolar, Universitat de València, España (jose.peirats@uv.es) \\ ${ }^{4}$ Departamento Didáctica y Organización Escolar, Universitat de València, España (angel.sanmartin@uv.es)
}

\section{RESUMEN:}

La influencia de la tecnología es una realidad innegable en todos los ámbitos de nuestra vida, por ello es fundamental el desarrollo de la competencia digital en la formación de postgrado de los futuros profesionales de la educación. Mucho se ha escrito en los últimos años sobre la formación docente en el uso de las tecnologías, y del análisis de los resultados obtenidos en investigaciones previas se constatan limitaciones en la formación específica en TIC recibida en los grados de educación en general, así como la tendencia al desarrollo de esta competencia desde una perspectiva transversal. Bajo esas premisas, en estas líneas se pretende describir y analizar una experiencia de aula basada en la introducción de las tecnologías de forma transversal en la asignatura de Procesos y Contextos Educativos del Máster de Profesorado de Educación Secundaria de la Universitat de València. Los participantes fueron 42 estudiantes, 25 hombres y 18 mujeres, que han cumplimentado un cuestionario de Google elaborado ad hoc. Sus resultados indican una valoración positiva del alumnado y el desarrollo de la competencia digital docente. También se desprende la posibilidad de desarrollar, transversalmente, la competencia digital docente en cualquier asignatura de los planes de estudios y la importancia de su abordaje desde la formación inicial del profesorado, con el objetivo de favorecer la implementación de buenas prácticas en su futuro profesional.

\section{PALABRAS CLAVE: COMPETENCIA DIGITAL, FORMACIÓN, DOCENTES, TRANSVERSALIDAD, MÁSTER.}

\section{ABSTRACT:}

The influence of technology is an undeniable reality in all areas of our life, which is why the development of digital competence in the postgraduate training of future professionals in education is fundamental. Much has been written in recent years on teacher training in the use of technologies, and the analysis of the results obtained in previous research shows limitations in the specific training in ICT received in general education degrees, as well as the tendency to develop this competence from a transversal perspective. Under these premises, these lines are intended to describe and analyze a classroom experience based on the introduction of ICT in a transversal way in the subject of Processes and Educational Contexts of the Master's Degree in Secondary Education at the University of Valencia. The participants were 42 students, 25 men and 18 women, who have completed a Google questionnaire developed ad hoc. Their results indicate a positive assessment of the students and the development of the digital teaching competition. It also reveals the possibility of developing, transversally, the digital teaching competence in any subject of the curricula and the importance of its development from teacher training, 
with the aim of favoring the implementation of good practices in their professional future.

KEYWORDS: DIGITAL COMPETENCE, TRAINING, TEACHERS, TRANSVERSALITY, MASTER.

\section{INTRODUCCIÓN}

En la sociedad del siglo XXI muchos de los cambios que se producen están fuertemente influenciados por una serie de innovaciones tecnológicas caracterizadas porque, invariablemente, van creciendo de forma exponencial. Consecuentemente, los avances tecnológicos inciden en mayor o menor grado en los aspectos más destacados de la sociedad, tales como la economía, la cultura, la política y también en la educación (Area y González, 2015; Zempoalteca, Barragán, González y Guzmán, 2017); y van marcando la vida de un ciudadano que debería estar preparado, con cierta mirada crítica, para poder ser competente en la utilización de estos avances científico-tecnológicos.

Actualmente, se comparte que la necesidad de formación de los ciudadanos se prolonga más allá de los primeros estudios y se extienden a lo largo de toda su vida (Domingo y Marquès, 2011). En este aspecto, la enseñanza formal $y$, en especial, el profesorado, sea cual sea el contexto, nivel o etapa en el que desarrolle su desempeño profesional, tiene un papel fundamental como facilitador de la aproximación a lo digital (Du Plessis y Webb, 2012; Roig y Pascual, 2012), por lo que se convierte en indispensable, tal y como apunta Marquès (2008), la formación didáctico-tecnológica de los futuros docentes.

Hoy en día, un profesional de la educación no puede ser un mero conocedor de la materia que imparte, sino que debe ser un agente capacitado para saber cómo gestionar la información de la que dispone y, como guía, administrarla y transmitirla a su alumnado. El docente deja de ser un experto en determinados conocimientos para ser un mediador de estos, competente en ofrecer los mejores medios por los que acceder a la información y orientar hacia un uso correcto por parte del alumnado. Según diversos autores, una verdadera adquisición de la competencia digital facilitaría la realización de estas tareas en gran medida (Esteve, González, Gisbert y Espuny, 2017; Gisbert, 2002; Roig y Pascual, 2012).

Sin embargo, a pesar del incremento de dotación tecnológica en los centros educativos, con mejoras en las infraestructuras y equipamiento en tecnologías de la información y la comunicación (TIC) y de la formación específica relacionada en este ámbito, las investigaciones señalan que la práctica educativa cotidiana no se ha modificado sustancialmente (Area, 2010; De Pablos, 2015; San Martín, Peirats y Gallardo, 2014). En esa dirección, distintos autores apuntan, entre sus causas, que los futuros maestros y maestras presentan insuficientes conocimientos sobre el uso de estas herramientas, que apenas poseen destrezas a nivel básico, que no están tan familiarizados con las TIC como se piensa $y$, principalmente, que ignoran su potencial didáctico, así como las posibles formas de integración en el currículo (Gutiérrez, 2014; Gutiérrez y Serrano, 2016; Losada, Valverde y Correa, 2013; Marcano, Íñigo y Sánchez, 2017; Peirats, Gallardo, San Martín y Cortés, 2015; Vílchez y Pérez, 2013). Por el contrario, no existen datos tan precisos sobre la situación del profesorado de secundaria, ya que la diversidad en esa etapa educativa es aún mayor si cabe, dada la especialización en sus materias.

$\mathrm{Si}$ centramos el punto de mira en el uso adecuado, en las buenas prácticas de las herramientas tecnológicas, surge el concepto de competencia digital, siendo esta una de las competencias clave que el alumnado y, previamente, el profesorado, debe adquirir para poder interactuar y desarrollarse en una sociedad fuertemente marcada por la tecnología (LOMCE, 8/2013). En este novedoso enfoque, la inclusión de la competencia digital en el sistema educativo ha alentado la incorporación de la formación en tecnología educativa en los planes de estudios del profesorado, en mayor o menor medida dependiendo de cada universidad, dando lugar a la denominada competencia digital docente. Esta se define en el marco común de competencia digital docente como "el uso creativo, crítico y seguro de las tecnologías de información y comunicación para alcanzar los objetivos relacionados con el trabajo, la empleabilidad, el aprendizaje, el tiempo libre, la inclusión y la participación en la sociedad" (Instituto Nacional de Tecnologías Educativas y de Formación del Profesorado, 2017, p. 9) y se caracteriza por su carácter multidimensional (Lázaro, Usart y Gisbert, 2019). Este amplio concepto necesariamente implica una formación profesional transversal sobre el uso de lo digital en el contexto educativo, y que dote al futuro profesorado de competencias instrumentales, organizativas, comunicativas, de búsqueda y gestión de información, didácticas-metodológicas, de presentación de información, investigativas y de privacidad y seguridad en la red (Peirats, Marín, Granados y Morote, 2018; Pozuelo, 2014; Trujillo y Raso, 2010; Villalustre y Del Moral, 2010). 
Sin embargo, el desarrollo de la competencia digital del profesorado no es una cuestión sencilla de abordar y se requiere un mayor esfuerzo del que se ha realizado hasta el momento. Es necesario considerar un nuevo punto de vista, en el que el docente, como ciudadano de esta sociedad altamente digitalizada, consiga ser capaz de alcanzar e integrar en el desempeño de su profesión la competencia digital en cada una de las dimensiones expuestas con anterioridad. Y, al mismo tiempo, que en el ejercicio de su práctica docente logre conjugar, desde un punto de vista tanto didáctico como crítico, los saberes pedagógicos y los referidos a las distintas materias curriculares con los conocimientos sobre el uso instrumental de las TIC (Cabero, Barroso y Llorente, 2015; Flores y Roig, 2016).

Por tanto, el desarrollo de la competencia digital docente es un desafío que precisa ser abordado en la formación inicial del profesorado ya que, hoy en día, se requieren formas de enseñar que sean coherentes con las nuevas formas de aprender que caracterizan al siglo XXI (De Saint Pierre, 2008; Gabarda, Rodríguez y Moreno, 2017). En esta misma línea, diversos autores (Brun, 2011; Flores, 2014; Silva, Miranda, Gisbert, Morales y Onetto, 2016; Vaillant, 2013) sostienen que los planes de formación de los futuros docentes deben considerar la integración e implementación efectiva de las TIC, ya que de este modo serán competentes, tanto en conocimientos pedagógicos y disciplinares como en la aplicación de las tecnologías a la educación.

Atendiendo a todo esto, evidentemente deberemos tener presente cómo formar a los docentes en relación con las competencias digitales $\mathrm{y}$, en ese sentido, no se trata de capacitarles únicamente para el uso instrumental de las TIC, sino de dotarles de habilidades para la práctica, para aplicar las estrategias adecuadas y para saber cómo integrar de forma adecuada las tecnologías en el currículo, y esto solo se consigue mediante la formación transversal (Bates 2001; Llorente, 2008). Como vemos, es necesaria una fuerte apuesta para preparar a los futuros docentes, de modo que sean competentes digitalmente en sus aulas y, no solo eso, sino que al mismo tiempo desarrollen un sentido crítico ante lo digital. Para ello, se trata de equilibrar o disminuir el problema de la brecha digital, definida por la OCDE (2018) como la distancia existente entre individuos o áreas en relación a sus oportunidades para acceder a las TIC, que acaba reflejando diferencias entre países y dentro de los mismos (Brun, 2011; SITEAL, 2014; Small y Vorgan, 2008; Zempoalteca, Barragán, González y Guzmán, 2017).
El lugar idóneo en el que emprender este cambio es, sin duda, la universidad. Especialmente en las facultades de educación, ya que en sus aulas se está formando, en estos momentos, la próxima generación de profesorado que, en un futuro no muy lejano, aplicará y desarrollará sus funciones con la ciudadanía del mañana (Cózar y Roblizo, 2014; Gallego, Gámiz y Gutiérrez, 2010; Roig y Pascual, 2012). Esto se justifica porque las competencias clave tienen gran relevancia en el ámbito educativo y en la vida. Pero, entre ellas, sin duda alguna, la vinculada con el tratamiento y uso de la información, es decir, la competencia digital, se ha convertido en una de las más relevantes para el profesorado del siglo XXI, al verificarse que esta capacitación es el factor clave para que se produzca el necesario cambio metodológico en las escuelas (Cózar y Roblizo, 2014; Flores y Roig, 2016; Gisbert 2002; Marquès 2008; Peirats, Muñoz y San Martín, 2015; Pozuelo, 2014; Prendes y Gutiérrez, 2013).

Teniendo en cuenta la relevancia de lo expuesto en las anteriores líneas y, con el fin de implementar en la práctica la argumentación conceptual desarrollada, se llevó a cabo una experiencia piloto en un aula universitaria durante un curso académico. Esta experiencia se centró en la introducción de tareas apropiadas para el desarrollo de la competencia digital docente en una asignatura troncal anual del Máster de Profesorado de Secundaria de la Universitat de València (UV).

Para finalizar este apartado, señalamos que el estudio realizado ha pretendido alcanzar los siguientes objetivos: a) analizar la percepción de competencia digital del alumnado participante; b) valorar los efectos de la intervención sobre la competencia digital docente y c) conocer la importancia dada a esta competencia para su futuro profesional.

\section{MÉTODO}

\subsection{Diseño}

Los datos que aquí presentamos son el resultado de un estudio descriptivo realizado en contexto natural con selección de la muestra a propósito (Patton, 2002) o también denominada muestra deliberada (Patton, 1990). Esta selección vino determinada, inicialmente, por la configuración de los gruposclase en el Máster de Profesorado de Secundaria de la Universitat de València. Los grupos se forman tomando como criterio la especialidad docente, en este caso la Música. Cabe destacar que se ha elegido una asignatura de este máster porque este postgrado 
está presente en las cuatro universidades públicas de la Comunidad Valenciana.

\subsection{Participantes}

En cuanto a los participantes, el grupo 19 de la asignatura de Procesos y Contextos Educativos está formado por 42 alumnos, 24 hombres (57\%) y 18 mujeres (43\%), de edades comprendidas entre los 22 y los 45 años, siendo la media de edad 25,43 años. De ellos, un 43\%, además de cursar el Máster de Profesorado de Secundaria, tiene otra ocupación remunerada. Respecto a su lugar de nacimiento se destaca que la mayoría (86\%) son nacidos en la Comunidad Valenciana (figura 1), contexto territorial caracterizado por la existencia de dos lenguas oficiales, castellano y valenciano, cuya competencia debe acreditarse antes de finalizar el máster, es decir, se considera requisito obligatorio para obtener el título.

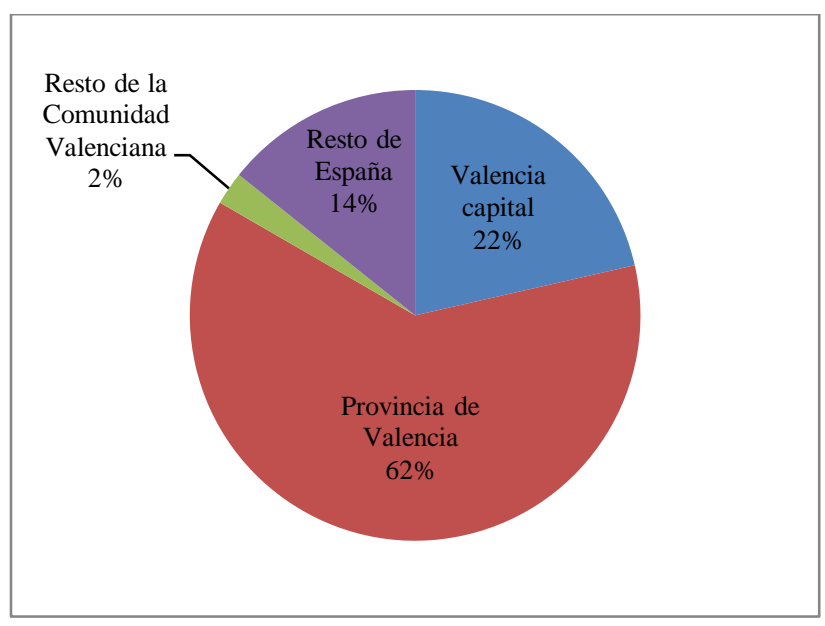

Figura 1. Lugar de nacimiento

\subsection{Instrumento}

El instrumento utilizado para la recogida de la información ha sido elaborado con la herramienta Google formularios. En él se preguntó sobre cuatro aspectos. El primero sobre las competencias clave que poseen, cuáles creen que van a desarrollar en el máster en general y en esta asignatura en particular, cuáles consideran fundamentales para desempeñar su labor docente y para la vida. En segundo lugar, se evalúa su autoeficacia en la competencia digital y competencia digital docente. El tercer aspecto se dedica a las actitudes hacia las TIC y cómo de importante consideran que es el desarrollo de esta competencia para su práctica docente futura. Por último, en cuarto lugar se les interrogó sobre los factores que influyen en la introducción de las TIC en las aulas y de qué depende que se produzca el cambio real en las prácticas de aula.

En este artículo se presentan los resultados de la segunda y tercera cuestión evaluada.

\subsection{Procedimiento}

Respecto al procedimiento se pueden diferenciar cinco fases que se describen a continuación.

Fase 1. Análisis de la realidad. En las primeras sesiones del curso se obtuvo información sobre el conocimiento y usos previos de tecnologías y la aplicación de estas herramientas en el ámbito educativo. Para conseguirlo, se empleó la observación y se preguntó al alumnado sobre sus preferencias, manejo, además de su competencia digital y competencia digital docente autopercibida.

Fase 2. Búsqueda de información. Posteriormente se propuso como tarea la búsqueda y el análisis de lo dispuesto sobre la competencia digital en el Decreto por el que se establece el currículo para Educación Secundaria en la Comunidad Valenciana (Decreto 87/2015 de la Generalitat Valenciana), para realizar un primer acercamiento al tema y concienciarles sobre la necesidad de desarrollar esta competencia.

Fase 3. Puesta en práctica. A lo largo de un curso (octubre-mayo) los alumnos participantes en esta experiencia realizaron su e-portfolio; prepararon presentaciones con PowerPoint o Prezzi; emplearon herramientas para gamificar la evaluación como Kahoot!, Socrative o Plickers; analizaron y utilizaron herramientas tecnológicas específicas para el campo de la Educación Musical como Musescore o Beatlab; seleccionaron recursos disponibles en Internet; cumplimentaron y organizaron sus entregas a través de un entorno virtual, como es el Aula Virtual de la UV, y dispusieron de tutorías virtuales durante todo el proceso.

Fase 4. Valoración de la experiencia. Al finalizar el curso el alumnado recibió por mail el enlace del cuestionario elaborado ad hoc para valorar el desarrollo de la competencia digital y la puesta en práctica de las actividades realizadas. La realización del cuestionario era anónima, voluntaria y, tal y como se les informó, no influía de ninguna manera en la nota final de la asignatura. Al cuestionario se pudo acceder durante quince días para poder abarcar a la gran diversidad de horarios de trabajo y exámenes del alumnado.

\subsection{Análisis de datos}

Una vez recopilada la información el análisis de los datos se realizó mediante el programa estadístico SPSS versión 20. 


\section{RESULTADOS}

Los resultados obtenidos se presentan, a continuación, siguiendo los objetivos marcados al final del primer apartado.

En cuanto al primero de los objetivos, centrado en analizar la percepción de competencia digital del alumnado participante, tras el análisis de la realidad, realizado en la fase 1 de esta experiencia, se destaca que la percepción del alumnado participante sobre su competencia digital al inicio de la asignatura se sitúa casi en un notable, con una media de 6.6 y que no todos se aprueban, aunque sí la gran mayoría (93\%). Un $7 \%$ se suspende con un 4 , un $14 \%$ se adjudica un aprobado, un $7 \%$ se califica con un bien, un $61 \%$ se autoevalúa con un notable y un $7 \%$ se sitúa en el sobresaliente. Por tanto, todo el alumnado se puntúa entre el 4 y el 9 en competencia digital.

Al finalizar el curso, la puntuación media de los participantes en cuanto a competencia digital ha aumentado ligeramente $(\overline{\mathrm{X}}=6.94)$, pero sigue $\sin$ llegar al notable. A pesar de que no se trata de un aumento significativo, se perciben diferencias en cuanto a la calificación en las autoevaluaciones (figura 2). Un 5\% se asigna un suspenso, un $17 \%$ se adjudica un bien, el $50 \%$ se sitúa en un notable y un $22 \%$ se califica con un sobresaliente.

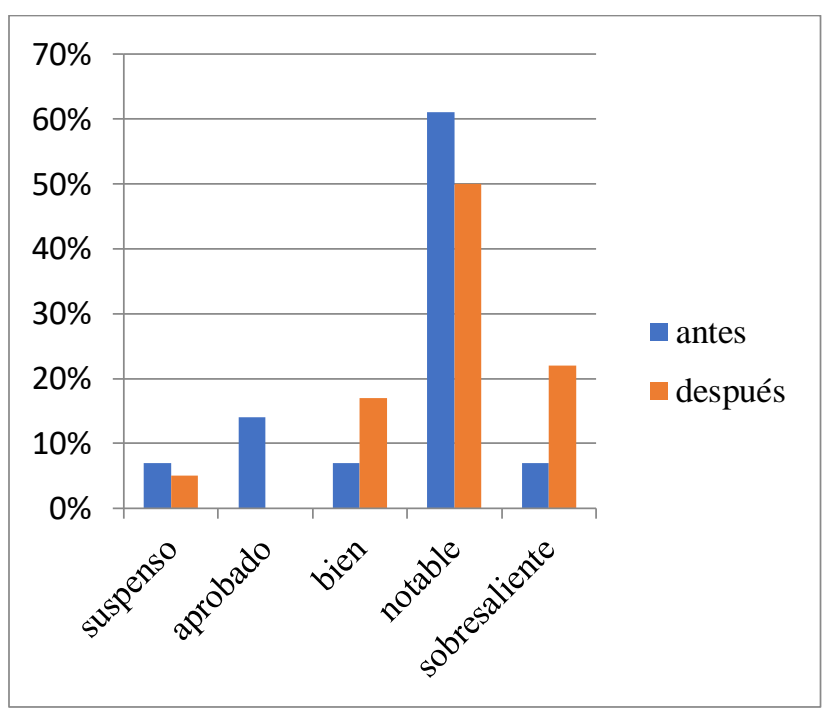

Figura 2. Autoeficacia en competencia digital

Por lo que respecta al segundo de los objetivos, focalizado en valorar los efectos sobre la competencia digital docente a través de la autopercepción del alumnado participante sobre su competencia digital docente, se entiende como la capacidad para la selección, uso y creación de herramientas y programas educativos digitales. Al inicio de la asignatura, la media del grupo se sitúa en un aprobado $(\overline{\mathrm{X}}=5.5)$. Un $25 \%$ se suspende, un $18 \%$ se aprueba, otro $18 \%$ se considera para un bien y el resto, un $39 \%$, se califica con un notable. Destaca que nadie, en el momento inicial, se puntúa con un sobresaliente y que un cuarto del total se sitúa por debajo del 5.

Pasado un curso, las autoevaluaciones de los participantes en cuanto a competencia digital docente son bien distintas (figura 3 ). La media del grupo ha aumentado hasta el notable $(\overline{\mathrm{X}}=7.2)$. Analizando las puntuaciones, destaca que no existe ningún estudiante que se asigne valores por debajo del bien. En esta puntuación se encuentra un $11 \%$, en el notable un $72 \%$ y en el sobresaliente un $10 \%$ del total de los encuestados. Destaca que al finalizar la experiencia todo el alumnado se concentra en puntuaciones altas, entre un 6 y un 10.

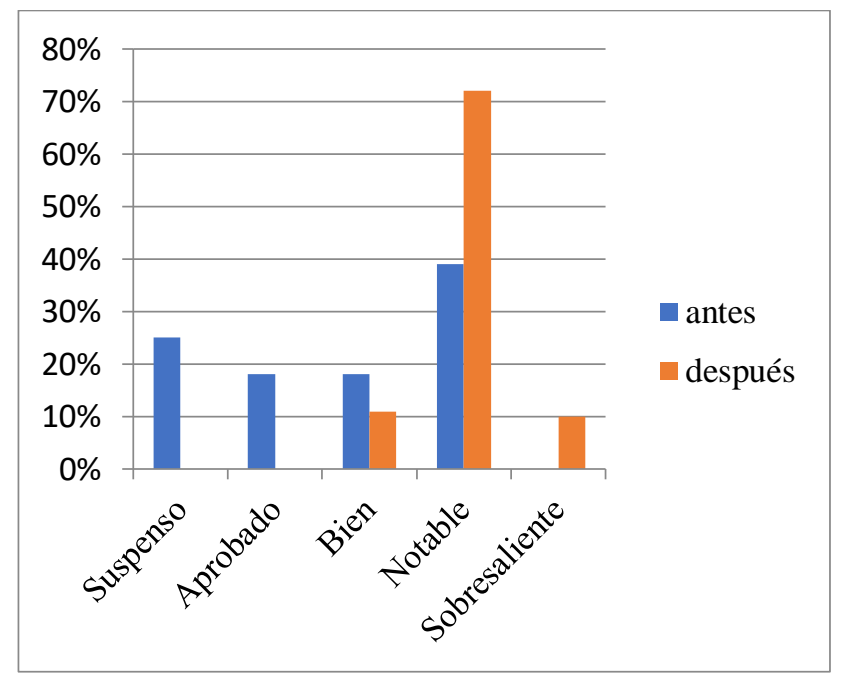

Figura 3. Autoeficacia en competencia digital docente

La diferencia entre la media del grupo, antes y después de la intervención con TIC en la competencia digital docente desde una perspectiva transversal, es estadísticamente significativa $(\mathrm{F}=18.18, \mathrm{p}<.01)$. Tal y como se muestra en la tabla 1, la diferencia de medias entre los dos momentos es notable y la dispersión de las puntuaciones en el momento de evaluación final es mucho menor. Esto indica que en el momento del postest las autoevaluaciones de los participantes están más próximas entre sí y cercanas a la media del grupo; por tanto, estamos ante una distribución homogénea y se considera que la media es un valor representativo. 
Tabla 1. Estadísticos descriptivos

\begin{tabular}{lcc}
\hline & Media & $\begin{array}{c}\text { Desviación } \\
\text { Estándar }\end{array}$ \\
Antes & 5.54 & 1.972 \\
Después & 7.25 & 0.79931 \\
\hline
\end{tabular}

Por último, con la realización de este estudio también se pretendía conocer la importancia dada por el alumnado participante a la competencia digital para su futuro profesional. La mayoría destaca que es bastante importante $(29 \%)$ o fundamental $(68 \%)$ en el momento inicial. Al finalizar el curso, la percepción de la mayoría del grupo sobre la importancia de esta competencia se sigue situando entre bastante importante $(23,5 \%)$ y fundamental $(58,58 \%)$, pero aumenta el porcentaje de alumnado que sitúa la importancia de esta competencia en la profesión docente en un nivel intermedio $(17,6 \%)$.

\section{DISCUSIÓN}

El desarrollo de las competencias clave hoy en día es una cuestión que preocupa enormemente, tanto a la administración, como a las universidades y a los docentes de todas las etapas educativas. Parece evidente que el lugar idóneo para iniciar su desarrollo y adquisición es la universidad (Díaz y Martín, 2015), ya que en sus aulas se están formando todos los docentes del mañana (Cózar y Roblizo, 2014; Gallego, Gámiz y Gutiérrez, 2010; Roig y Pascual, 2012).

En este estudio hemos centrado nuestro interés en la competencia digital, por las implicaciones prácticas en la educación de una sociedad que Castells (1997) hace tiempo denominó como la sociedad red, y mucho antes McLuhan (1967) como aldea global. Una sociedad, hoy, altamente digitalizada en todos sus ámbitos, no solo el educativo, en la que conviven unos niños y jóvenes, a los que hay que ofrecer una adecuada formación tecnológica.

La competencia digital es una de las competencias clave y se debe adquirir en la educación básica; por ello, los docentes han de tener un alto nivel de capacitación en competencia digital (Rodríguez, Martínez y Raso, 2017), ya que ellos son los encargados de su desarrollo en las futuras generaciones (Rodríguez, Cáceres y Alonso, 2018), que son los ciudadanos del mañana (Gisbert y Lázaro, 2015). En esta línea, Rodríguez, Raso y Ruiz (2019) recientemente han realizado un metaanálisis centrado en la competencia digital y la formación del profesorado y concluyen la relevancia del tema y el aumento de la producción científica en los últimos años.

Si nos centramos en la Comunidad Valenciana, de las cuatro universidades públicas en las que se imparte el Máster de Profesorado de Secundaria, solo una de ellas, la Universidad Miguel Hernández de Elche, cuenta con una asignatura troncal de formación obligatoria relacionada directamente con las TIC, denominada "Tecnologías de Información y Comunicación aplicadas a docencia y aprendizaje en enseñanza secundaria". Esta realidad nos indica que la política de la mayoría de las universidades de nuestra comunidad, al igual que en el resto del territorio español (Peirats, Marín, Morote y Granados, 2018), están apostando por la formación en competencia digital del profesorado de las diferentes etapas educativas, como Infantil (Aristizabal y Cruz, 2018), Primaria, Secundaria y Formación Profesional, desde una perspectiva transversal.

De acuerdo con estudios recientes (Brun, 2011; Flores, 2014; Silva et al., 2016; Vaillant, 2013) señalamos que es necesario que los planes de formación de los futuros docentes consideren específicamente la integración e implementación efectiva de las TIC, independientemente de variables como el sexo o la edad (Hernández y Torrijos, 2019). Es más, el objetivo final ha de ser capacitar para la práctica y, de acuerdo con Bates (2001) o Llorente (2008), esto solo se consigue mediante la formación transversal.

Los resultados obtenidos tras la experiencia de un curso de integración transversal de las TIC han sido prometedores e indican, como en otros estudios (Islas, 2018), que las mejoras se producen cuando la tecnología se emplea para compartir información, acceder a recursos o emplear aplicaciones para la resolución de tareas desde una perspectiva crítica. En este caso se ha encontrado un aumento significativo en la percepción de eficacia del alumnado participante respecto a su competencia digital docente, lo que nos lleva a considerar que la transversalidad es una alternativa apropiada para la mejora de la formación tecnológica de nuestro futuro profesorado.

\section{CONCLUSIONES}

En los últimos tiempos, con los nuevos planes de estudio de grados y máster relacionados con la educación, ha aumentado la preocupación sobre un tema complejo y actual, focalizado en torno a si los estudiantes y futuros docentes reciben o no una formación adecuada en cuanto al conocimiento y uso 
de tecnologías digitales (Aguaded, 2009; Herrada y Herrada, 2011).

Al hilo de ese discurso, hemos querido realizar un estudio en el que se comprueba que los datos de la línea base son claramente inferiores a los obtenidos tras un curso académico trabajando la competencia digital en el aula de forma transversal, especialmente cuando se centra la atención en la propia percepción de los participantes sobre la competencia digital docente adquirida.

Por otra parte, el trabajo transversal, que en este caso hemos presentado, ha demostrado mejorar la competencia digital docente, aunque la formación en TIC en esta modalidad se deja a la voluntad del profesorado que imparte la asignatura en cuestión y que, necesariamente, debe ser competente tanto en los contenidos de la asignatura como en la integración y aplicación curricular de las TIC en la misma. Solo de esta forma se conseguirá que el impacto de las tecnologías se vea reflejado en innovaciones pedagógicas, en las que prime la perspectiva didáctica sobre la tecnológica, y que repercuta en la mejora de la calidad de la institución escolar (Gisbert y Lázaro, 2015).

Por tanto, para que la formación transversal en competencia digital sea completa y pueda reflejarse en buenas prácticas mediadas con TIC, debe ser asumida y realizada por la mayor parte del profesorado de la propia titulación; en caso contrario, se puede correr el riesgo de realizar una excelente formación transversal, pero de forma parcial o fragmentaria en la formación inicial global de las próximas generaciones de docentes.

Finalmente, este resultado parece reforzar la necesidad de introducir el desarrollo de competencias, como la digital, en los planes de estudio de los futuros profesionales de la educación, especialmente profesorado de Primaria, Secundaria y Formación Profesional, volviendo al modelo anterior en el que se contemplaba una asignatura troncal, sin abandonar el enfoque transversal desde otras materias. De esta forma, se conseguiría que los docentes del mañana incorporasen de forma natural herramientas y recursos tecnológicos en sus prácticas docentes para mejorar los procesos de enseñanza-aprendizaje (Marcano, Íñigo y Sánchez, 2017).

\section{LIMITACIONES DEL ESTUDIO}

Una de las limitaciones de este estudio se relaciona con el tamaño y la selección de la muestra. Es necesario destacar que el número de participantes del estudio es reducido $(n=42)$, por lo que puede considerarse insuficientemente significativa para obtener resultados generalizables.

Asimismo, esta muestra no ha sido seleccionada mediante procedimientos aleatorios, debido a que quedó limitada al contexto educativo en el que fueron recogidos los datos. Esto supone que podría contener algún tipo de sesgo por tratarse de grupos formados por especialidades, en este caso música. Del mismo modo, cabe considerar que la experiencia ha podido llevarse a cabo únicamente en una asignatura del Máster, lo que implica que los resultados quedan limitados a una asignatura de todo el plan de estudios. Sería interesante comprobar, en futuros estudios, los resultados obtenidos al ampliar el número de materias implicadas y de grupos.

Por otra parte, debemos tener en cuenta que no se ha obtenido información de un grupo control, lo que podría haber sido de gran utilidad para comparar los datos y analizar las diferencias existentes entre ambos, así como la verdadera incidencia de la intervención llevada a cabo.

\section{PROSPECTIVA}

Por lo que respecta a las líneas de investigación futuras, tras la realización de este estudio surgen nuevas preguntas de investigación como, por ejemplo: ¿qué habría sucedido si se hubiese implementado esta metodología a más grupos el máster?, ¿qué diferencias habría habido en los resultados si se hubiese realizado la experiencia en el mismo grupo, pero desde distintas asignaturas?, ¿es la transversalidad, en definitiva, una opción eficaz para el desarrollo de competencias?...

Además, cabe destacar que este trabajo puede ser útil para ser conscientes de la necesidad de realizar una formación específica en competencia digital o para contribuir al debate sobre el enfoque transversal de inclusión de la formación de competencias en los planes de estudios. En esa dirección, en estas líneas se ha pretendido acercarnos al proceso de toma de decisiones y el diseño de tareas que permiten desarrollar la competencia digital, con la finalidad de responder adecuadamente a las necesidades de un alumnado que, no lo olvidemos, son los futuros docentes de nuestros centros educativos.

\section{REFERENCIAS}

Aguaded, I. (2009). Miopía en los nuevos planes de formación de maestros en España: ¿Docentes analógicos o digitales? Comunicar, 17(33), 7-8.

Area, M. (2010). El proceso de integración y uso pedagógico de las TIC en los centros educativos. Un estudio de casos. Revista de Educación, 352, 77-97. 
Area, M., y González, C. S. (2015). De la enseñanza con libros de texto al aprendizaje en espacios online gamificados. Educatio Siglo XXI, 33(3), 15-38.

Aristizabal, P., y Cruz, E. (2018). Desarrollo de la competencia digital en la formación inicial del profesorado de educacion infantil. Píxel-Bit. Revista de Medios y Educación. Píxel-Bit. Revista de Medios y Educación 52(1), 97-110. doi: http://dx.doi.org/10.12795/pixelbit.2018.i52.07

Bates, T. (2001). Afrontar el reto tecnológico en los centros universitarios e institutos. En T. Bates (Ed.), Cómo gestionar el cambio tecnológico (pp. 25-56). Barcelona: Gedisa.

Brun, M. (2011). Las tecnologías de la información y las comunicaciones en la formación inicial docente de América Latina. Serie Políticas Sociales, 172(9), 10-73.

Cabero, J., Barroso, J., y Llorente, M. C. (2015). E-actividades para la formación del profesorado en tecnologías de la información y comunicación en el Proyecto Dipro 2.0. Sevilla: Grupo de Investigación Didáctica de la Universidad de Sevilla. Recuperado de http://go.uv.es/M1JoM2p

Castells, M. (1997). La era de la información: economía, sociedad y cultura. Volumen I: La sociedad Red. Madrid: Alianza Editorial.

Cózar, R., y Roblizo, M. J. (2014). La Competencia Digital en la formación de los futuros maestros: percepciones de los alumnos de los Grados de Maestro de la Facultad de Educación de Albacete. RELATEC, Revista Latinoamericana de Tecnología Educativa, 13(2), 119133.

De Pablos, J. (coord.). (2015). Los centros educativos ante el desafío de las tecnologías digitales. Madrid: La Muralla

De Saint Pierre, D. (2008). Futuros profesores: ¿por qué formar en competencias digitales? Chile: Enlaces. Ministerio de Educación. Recuperado de http://goo.gl/2nYaew

Decreto $87 / 2015$, de 5 de junio, del Consell, por el que establece el currículo y desarrolla la ordenación general de la Educación Secundaria Obligatoria y del Bachillerato en la Comunitat Valenciana (DOCV 7544 de 10.06.2015).

Díaz, M. D., y Martín, J. J. (2015). Evaluación del perfil competencial de alumnos universitarios respecto a la educación tecnológica. Innoeduca. International Journal of Technology and Educational Innovation, 1(1), 17-24. doi:10.20548/ijtei.v1i1.29

Domingo, M., y Marquès, P. (2011). Aulas 2.0 y uso de las TIC en la práctica docente. Comunicar, 19(37), 169-175. doi:10.3916/C37-2011-03-09

Du Plessis, A., y Webb, P. (2012). A teacher proposed heuristic for ICT professional teacher development and implementation in the south African context. Turkish Online Journal of Educational Technology, 11(4), 4655. Recuperado de http://go.uv.es/Oz5X1NX

Esteve, F., González, J., Gisbert, M., y Espuny, C. (2017). Los graduados universitarios y su Competencia Digital. En A. C. Chumaceiro, F. Ramos, y J. Hernández (Eds.), Procesos formativos para el siglo XXI (pp. 5675). Cabimas: Fondo Editorial UNERMB.

Flores, C. (2014). Competencia Digital Docente: desempeños didácticos en la formación inicial del profesorado. Revista científica de educación y comunicación. Hachetetepe, 9, 55-70.

Flores, C., y Roig, R. (2016). Percepción de estudiantes de Pedagogía sobre el desarrollo de su Competencia Digital a lo largo de su proceso formativo. Estudios Pedagógicos, 42(3), 129-148. doi:10.4067/S071807052016000400007
Gabarda, V., Rodríguez, A., y Moreno, M. D. (2017). La Competencia Digital en estudiantes de magisterio. Análisis competencial y percepción personal del futuro maestro. Educatio Siglo XXI,35(2), 253-274. doi: $10.6018 / \mathrm{j} / 298601$

Gallego, M. J., Gámiz, V., y Gutiérrez, E. (mayo de 2010). Competencias digitales en la formación del futuro docente. Propuestas didácticas. En J. Pérez, J. Cabero y L. Vilches (dirección), I Congreso EuroIberoamericano de Alfabetización Mediática y Culturas Digitales. Sevilla: Universidad de Sevilla.

Gisbert, M. (2002). El nuevo rol del profesor en entornos tecnológicos. Acción Pedagógica 11(1), 48-59.

Gisbert, M., y Lázaro, J. L. (2015). Professional development in teacher digital competence and improving school quality from the teachers' perspective: a case study. New approaches in educational research, 4(2), 124-131. doi:10.7821/naer.2015.7.123

Gutiérrez, I. (2014). Perfil del profesor universitario español en torno a las competencias en tecnologías de la información y de la comunicación. Pixel-Bit. Revista de Medios y Educación, 44, 51-65. doi:10.12795/pixelbit.2014.i44.04

Gutiérrez, I., y Serrano, J. L. (2016). Evaluation and development of digital competence in future primary school teachers at the University of Murcia. Journal of new Approaches in Educational Research, 5(1), 53-59. doi:10.7821/naer.2016.1.152.

Hernández, J. P., y Torrijos, P. (2019). Percepción del profesorado universitario sobre la integración de las Tecnologías de la Información y la Comunicación (TIC) en las modalidades docentes. Influencia del género y la edad. Edmetic 8(1), 128-146.

Herrada, R., y Herrada, G. (2011). Adaptación de los estudios de magisterio al EEES: Las TIC en los nuevos planes de estudio, Edutec-e. Revista electrónica de Tecnología Educativa, 36, 1-12. doi:10.21556/edutec.2011.36.405

Instituto Nacional de Tecnologías Educativas y de Formación del Profesorado (2017). Marco común de Competencia Digital Docente. Madrid: Ministerio de Educación. Recuperado de: goo.gl/7pvLve

Islas, C. (2018). Implicación de las TIC en el aprendizaje de los universitarios: una explicación sistémico-conectivista. Pixel-Bit, 52, doi:10.12795/pixelbit.2018.i52.14

Lázaro, J. M., Usart, M., y Gisbert, M. (2019). Assessing Teacher Digital Competence: the Construction of an Instrument for Measuring the Knowledge of PreService Teachers. Journal of New Approaches in Educational Research 8(1). doi:10.7821/naer.2019.1.370

Losada, D., Valverde, J., y Correa, J. M. (2013). La tecnología educativa en la universidad pública española. Pixel-Bit. Revista de Medios y Educación, 41, 133-148.

Llorente, M. (2008). Aspectos fundamentales de la formación del profesorado en TIC. PixelBit: Revista de medios y educación, 31, 121 -130.

Marcano, B., Íñigo, V., y Sánchez J. M. (2017). Percepción personal de una actividad de creación de contenido colaborativo de estudiantes del Máster de Formación del $\begin{array}{lll}\text { Profesorado. } & \text { EDMETIC, } & 6(2), \\ \end{array}$ doi:10.21071/edmetic.v6i2.6932

Marquès, P. (2008). Impacto de las TIC en la enseñanza universitaria. Didáctica, Innovación y Multimedia, 11, 1-15. Recuperado de http://go.uv.es/IercV0s 
McLuhan, M. (1967). The Gutenberg Galaxy: The Making of Typographic Man. Toronto: University of Toronto Press.

Ministerio de Educación, Cultura y Deporte (2013). Ley Orgánica 8/2013, de 9 de diciembre, para la Mejora de la Calidad Educativa. Boletín Oficial del Estado, nº 295. Recuperado de http://go.uv.es/74m4UYJ

OCDE (2018). Regards sur l'éducation. Les indicateurs de L'OCDE, Paris: Éditions OCDE. doi:10.1787/eag-2018fr

Patton, M. Q. (1990). Qualitative Evaluation and Research Methods ( $\left.2^{\text {nd }} e d.\right)$. Newbury Park, California: Sage Publications.

Patton, M. Q. (2002). Qualitative research and evaluation methods $\left(3^{r d} e d.\right)$. Thousand Oaks, California: Sage Publications.

Peirats, J., Gallardo, I. M., San Martín, A., y Cortés, S. (2015). Los contenidos curriculares digitalizados: Voces y silencios en el ámbito editorial. Educatio Siglo XXI, 33(3), 39-62. doi:10.6018/j/240801

Peirats, J., Marín, D., Granados, J., y Morote, D. (2018). Competencia digital en los planes de estudios de universidades públicas españolas. REDU. Revista de Docencia Universitaria, 16(1), 175-191.

Peirats, J., Muñoz, J. L., y San Martín, Á. (2015). Los imponderables de la Tecnología Educativa en la formación del profesorado. RELATEC, Revista Latinoamericana de Tecnología Educativa, 14(3), 1122. doi:10.17398/1695-288X.14.3.11

Pozuelo, J. (2014). ¿Y si enseñamos de otra manera?: competencias digitales para el cambio metodológico. Caracciollos: Revista digital de investigación en docencia 2(1), 1-21. Recuperado de http://go.uv.es/n6bf7oH

Prendes, M. P., y Gutiérrez, I. (2013). Competencias tecnológicas del profesorado en las universidades españolas. Revista de Educación, 361, 196-222. doi:10.4438/1988- 592X-RE-2011-361-140

Rodríguez, A. M., Raso, F., y Ruiz, J. (2019). Competencia digital, educación superior y formación del profesorado: un estudio de meta-análisis en la Web Of Science. Píxel-Bit. Revista de Medios y Educación 54(1), 65-81. doi:10.12795/pixelbit.2019.i54.04

Rodríguez, A. M., Cáceres, Ma . P., y Alonso, S. (2018). The digital competence of the future teacher: bibliometric analysis of scientific productivity indexed in Scopus. IJERI. International Journal of Educational Research and Innovation, 10, 317-333.

Rodríguez, A. M., Martínez, N., y Raso, F. (2017). La formación del profesorado en competencia digital: clave para la educación del siglo XXI. Revista Internacional de Didáctica y Organización Educativa, 3(2), 46-65.

Roig, R., y Pascual, A. (2012). Las competencias digitales de los futuros docentes. Un análisis con estudiantes de Magisterio de Educación Infantil de la Universidad de Alicante. @ tic. revista d'innovació educativa, 9, 53-60.

San Martín, A., Peirats, J., y Gallardo, I. M. (2014). Centros educativos inteligentes. Luces y sombras sobre las políticas de transferencia de tecnología y las prácticas docentes. Profesorado. Revista de currículum $y$ formación del profesorado, 18(3), 63-79.

Silva, J., Miranda, P., Gisbert, M., Morales, J., y Onetto, A. (2016). Indicadores para evaluar la Competencia Digital Docente en la formación inicial en el contexto ChilenoUruguayo. RELATEC, Revista Latinoamericana de Tecnología Educativa, 15(3), 55-67.
SITEAL (2014). Informe sobre tendencias sociales y educativas en América Latina 2014. Recuperado de http://go.uv.es/WBoBrP3

Small, G. W., y Vorgan, G. (2008). El cerebro digital: cómo las nuevas tecnologías están cambiando nuestra mente. Barcelona: Ediciones Urano.

Trujillo, J. M., y Raso, F. (2010). Formación inicial docente y Competencia Digital en la convergencia europea (EEES). Enseñanza y Teaching, 28(1), 49-77.

Vaillant, D. (2013). Integración de TIC en los sistemas de formación docente inicial y continua para la Educación Básica en América Latina. Argentina: Fondo de las Naciones Unidas para la Infancia (UNICEF).

Vílchez, J., y Pérez, F. (2013). Percepción de Futuros Maestros sobre el potencial de las TIC en la Educación: de las Expectativas a la Realidad. Revista Fuentes, 13, 155172.

Villalustre, L., y Del Moral, M. (2010). Innovaciones didácticometodológicas en el contexto virtual de Ruralnet y satisfacción de los estudiantes universitarios. REICE. Revista Iberoamericana Sobre Calidad, Eficacia y Cambio en Educación, 8(5), 69-81.

Zempoalteca, B., Barragán, J. F., González, J., y Guzmán, T. (2017). Formación en TIC y Competencia Digital en la docencia en instituciones públicas de educación superior. Apertura, 9(1), 80-96. Recuperado de http://go.uv.es/ewV7He0 